\title{
Construction of Evaluation Model of University Student Education Evaluation System and Its MATLAB Simulation Based on BP Neural Network Algorithm*
}

\author{
Fen Yang ${ }^{1}$ \\ Henan University
}

\begin{abstract}
For BP neural network, it is easy to fall into a local optimum during the learning process. Using an improved algorithm to optimize its weights and thresholds, a classroom teaching quality evaluation model based on an improved-BP neural network is constructed, apply this algorithm to the actual teaching quality evaluation in a certain university, experiments show that this model can objectively evaluate the quality of classroom teaching comprehensively.
\end{abstract}

\section{Keywords}

Neural Network • Fuzzy Comprehensive Evaluation • Genetic Algorithm • Teaching Quality Evaluation

\footnotetext{
* This work is supported by the National Natural Science Foundation (No. 71371172) and 2017 Scientific Research Projects of Henan Province (No. 172102210523).

${ }^{1}$ Correspondence to: Fen Yang (MA), Henan University of Animal Husbandry \& Economy, Zhengzhou 450004, China. Email: 147735389@qq.com 
Evaluation of teaching quality is an important part of teaching management in institutions of higher learning. Its fundamental purpose is to master the actual teaching ability and teaching level of university teachers. However, most traditional evaluation methods use mathematical models that directly establish evaluation systems, such as the analytic hierarchy process, cluster analysis, grey system, fuzzy comprehensive evaluation method, etc., but these methods are difficult to eliminate all kinds of randomness and subjectivity in the evaluation process, and can easily cause distortion and bias in the evaluation results. In order to overcome artificial subjective arbitrariness, the evaluation results are more accurate and effective. By finding the mathematical relationship between input and output in a suitable evaluation system, a reasonable and scientific mathematical model is established, which will have an important significance for the evaluation of teaching quality (Zeng, Li, Song, Sheng \& Zhu, 2017).

Neural network is a nonlinear adaptive dynamic system composed of a large number of processing units. It has learning ability, memory ability, computation ability, and intelligent processing function. It simulates the information processing mechanism of the brain at different levels. It can be used for various data processing occasions such as prediction, classification, pattern recognition and process control. Compared with traditional data processing methods, it is more suitable for dealing with fuzzy, nonlinear problems and problems with ambiguous patterns. This paper applies the artificial neural network theory into the teaching quality evaluation of college teachers, presents a quantitative evaluation method based on three layers of neurons. Through the learning of known samples, it obtains experience, knowledge, and subjective judgment of the evaluation experts, as well as the ability to coordinate the weights of the importance of goals, then the network can reproduce the experience, knowledge, and intuitive thinking of the evaluation experts, thereby reducing the impact of human factors in the evaluation process, and fundamentally overcoming the difficulties in modelling and solving during the teaching quality evaluation processes. The method has the advantages of short time, high speed, and high accuracy, which well guarantees the objectivity of the evaluation results and provides a more meaningful reference value for the study of teaching quality evaluation system (Zhang \& Shen, 2009).

\section{Improved BP neural network}

\section{Basic principle of BP neural network}

BP neural network (Bai, Li, Feng \& Guo, 2010) also called multi-layer feed-forward neural network, consists of several layers of neurons. They can be divided into input layer, hidden layer and output layer. The feature of this neural network model is that each layer of neurons only has connections with neurons in adjacent layers; there is no connection between the neurons in each layer; there is no feedback connection between neurons in each layer.

The basic principle of the BP algorithm is the principle of error correction, which is to use the gradient descent method, through the back-propagation of network output error to adjust and modify the connection weights of the network so that the error is minimized (Guo, Gao, Yang, \& Kang, 2009; Tinto, 1975; Rebuffoscheer, Schmitt \& Scherer, 2007). Its learning process includes two stages: forward calculation and error back-propagation. When a network is given an input mode, it is passed from the input layer unit to the 
hidden layer unit, processed by the hidden layer unit, and then sent to the output layer unit, after processed by the output layer unit, an output response is generated (Sun, Bai, Geng \& Shi, 2017). If the error between the output response and the expected output does not meet the requirements, the error is returned layer by layer along the connection path and the connection weights and thresholds of the layers are corrected so that the error is reduced. After modifying the connection weights and thresholds, new connection weights and thresholds are used for calculating and processing the input mode to generate an output response to compare with the expected output, after repeated iteration and calculation, it stops until the error is less than a given value.

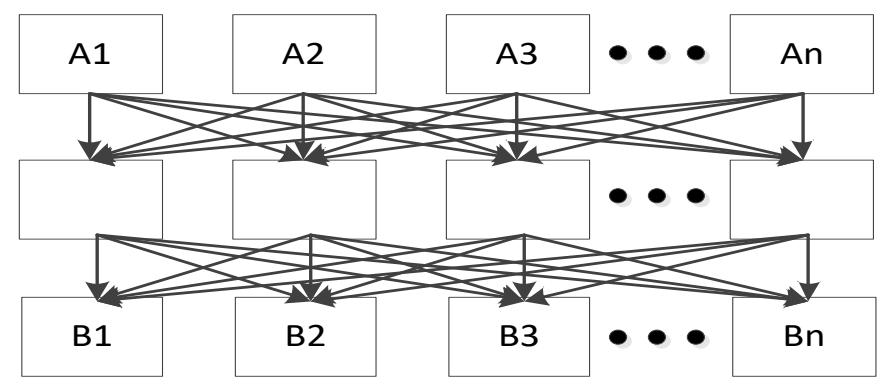

Figure 1. BP network topology.

\section{Execution steps of improved BP neural network}

(1) Initialization of parameters (Croft, Danson, Dawson, \& Ward, 2001): determine the number of input and output $\mathrm{N}, \mathrm{O}$, determine the number of layers of the network $\mathrm{L}$; set the population scale as $\mathrm{P}$, randomly generate a certain number of gene individuals $\mathrm{S} 0$, the algorithm's crossover probability is $\mathrm{Pc}$, mutation probability is $\mathrm{Pm}$, evolutionary generation number is $\mathrm{k}$;

(2) Define the fitness function $\mathrm{f}(x)=\sum_{i=1}^{N} \sum_{j=1}^{o}\left(Y_{i}(t)-Y_{j}(t)^{2}\right)$, where $\mathrm{Y}_{\mathrm{i}}(\mathrm{t})$ and $\mathrm{f}(\mathrm{x})$ are the actual output and expected output of the training data $t$ at the $\mathrm{i}$-th output node, respectively;

(3) Calculate the individual fitness function $\mathrm{f}(\mathrm{x})$ in the population;

(4) In accordance with genetic strategies, use selection, crossover, and mutation operators to act on groups to form the next generation of groups;

(5) Determine whether the feature of a group meets a certain indicator, or a predetermined number of iterations is achieved, if it is satisfied, turn to step (6), else go to step (3);

(6) Decode the best individuals in the population in coding order as the initial weights and thresholds of the BP network.

(7) Conduct forward-propagation of the BP network, calculate the global error, and judge whether it meets the requirements, and end the network learning if it is in compliance;

(8) If it is less than the loop count, perform BP network back-propagation, modify the threshold, and return to step (6), or end the network learning. 


\section{An Improved Learning Algorithm for Teaching Evaluation System}

The BP neural network model used for the evaluation of students' comprehensive quality is shown in Figure 1:

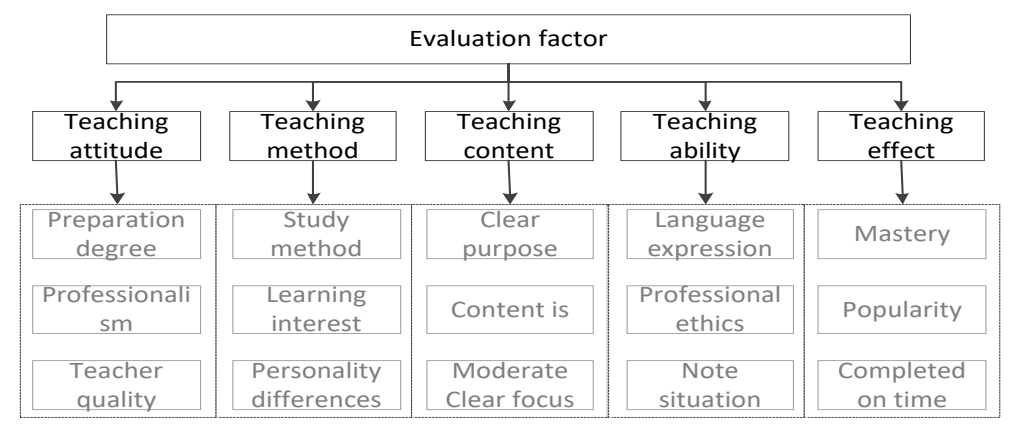

Figure 2. Teaching evaluation system.

\section{Initialize network parameters}

Determine the number of hidden layer nodes, the selection of the number of hidden layer nodes related to the accuracy of the entire BP network and learning efficiency (Wang, Shen, \& Xing, 2014). The input layer transfer function adopts hyperbolic tangent function tansig, and the transfer functions of hyperbolic hidden layer and output layer respectively use tansig and purelin, the training method used in this paper adopts LevenbergMarquardt, as shown in Figure 2.

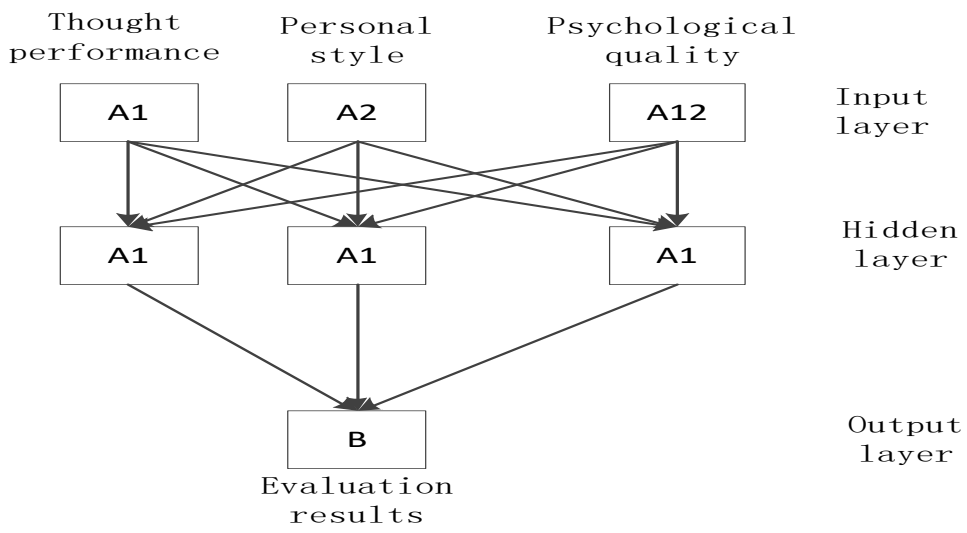

Figure 3. Comprehensive quality evaluation model. 


\section{Provide training samples}

The training samples are randomly sorted, and one pair of sample training data is selected in order, $\mathrm{x}$ is the input data of the input layer, py is the expected output, and $\mathrm{p}$ is the sequence number of the sample.

\section{Calculate output and error}

The output of the output layer neuron $\mathrm{k}$ is:

$$
y_{k}(p)=\frac{1}{1+e^{-v_{k}(p)}}
$$

Under the $\mathrm{p}$-th training sample, define the target cost function as:

$$
E=\frac{1}{2} \sum_{k=1}^{m} \sum_{p=1}^{L} y_{k}(p)-t_{k}(p)
$$

Where $\mathrm{L}$ is the number of tested samples, $\mathrm{m}$ is the number of neurons in the output layer. Set an allowable error $=0.01$, if $\mathrm{E}<\varepsilon$ or it reaches the maximum number of the set training times or the absolute rate of the changing of $E$ in each round is small enough, then end the learning, otherwise continue to the next step.

\section{Comprehensive Quality Evaluation Model Implementation of Evaluation Model Based on MATLAB}

\section{Data collection}

There is usually a certain error between the BP network trained by Matlab and the exact value. One common method of reducing errors is to perform a large number of simulations and select a better set of results. This method is relatively time consuming. Another method is to fine-tune the results of the existing network training results that are similar to the obtained results. Matlab software provides a powerful Simulink tool that can help regulate various parameters in a specific neural network and is quite easy to implement.

\section{Network test and result analysis}

After the neural network model is trained, in MATLAB, the four groups of data from 16 to 19 are normalized using the tramnmx function and then used for testing the model. The test results are compared with the expert evaluation results after being denormalized by the postmnmx function. Through comprehensive analysis of the data, the results proved to be satisfactory, indicating that the network training is successful and has a good generalization ability. 


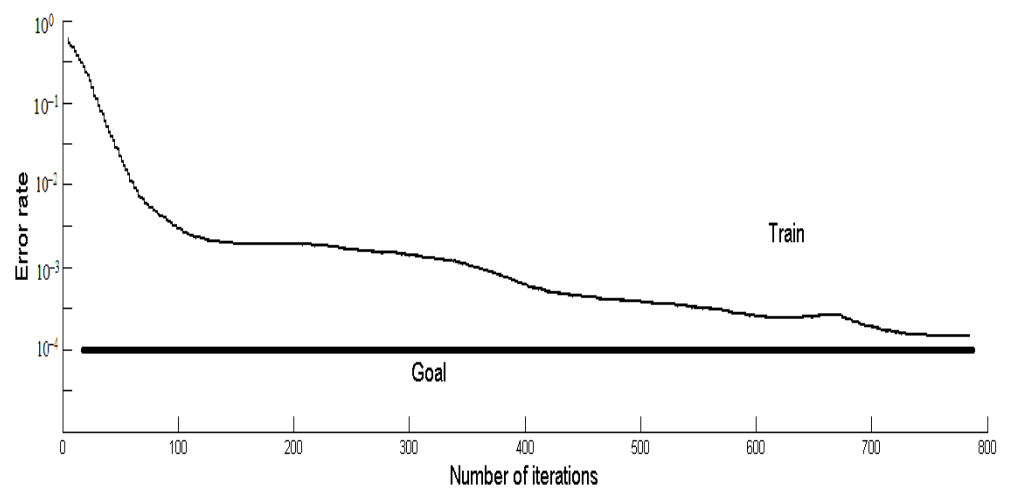

Figure 4. Sample training results.

At first, in the design of teacher's teaching quality evaluation method, input sample $\mathrm{X}$ into the network, the network obtained a score of 79.7411 for the teacher's teaching quality, and an error of $0.5753 \%$ for the fuzzy judgement result of 79.285. For the trained neural network, 800 samples were randomly used to simulate the network, and the 800 groups of data were evaluated using fuzzy evaluation rules, as shown in Figure 5. Considering that the input data in the actual application process generally conforms to specific structural features, it is believed that the neural network model has certain reliability in the evaluation of teaching quality, and the calculation is simple and fast.

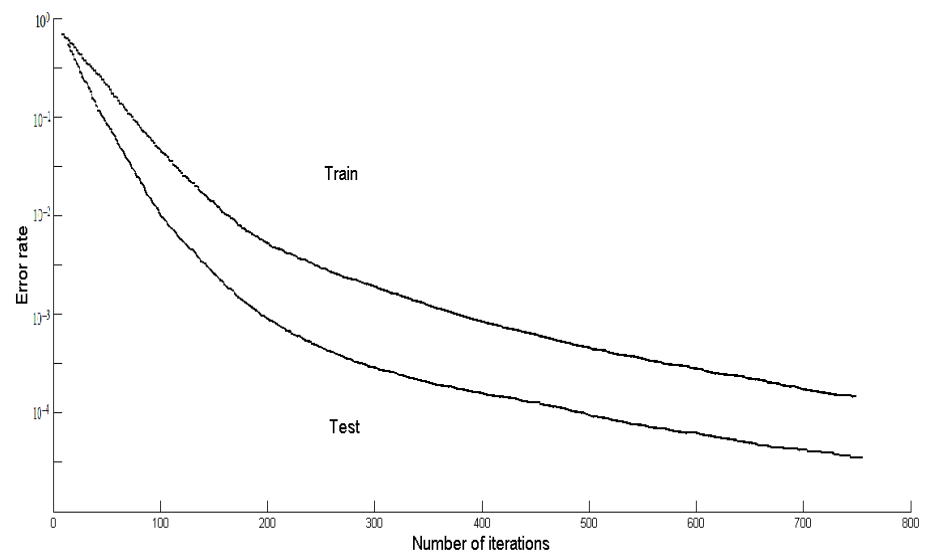

Figure 5. Sample training contrast.

After using the improved algorithm to optimize the weights and thresholds of the BP neural network, the accuracy of the algorithm is significantly improved with the increase in the number of iterations, see Figure 6 for details, it is proved that the improved algorithm is successful for the weight and threshold optimization of BP neural network. 


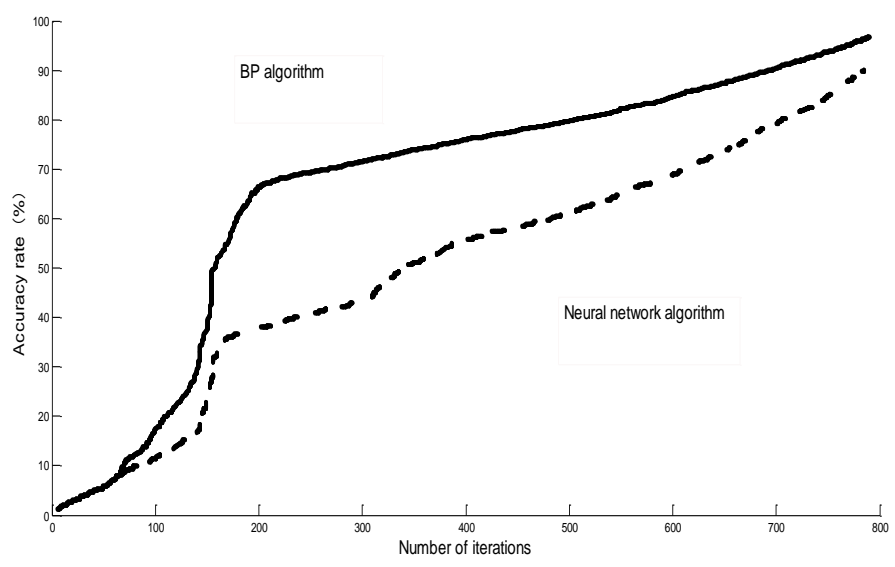

Figure 6. Comparison of accuracy.

\section{Conclusion}

The ultimate goal of college classroom teaching quality evaluation is to deepen teaching reform and improve teaching quality. The above research results show that it is feasible to use BP neural network for the evaluation of college students' comprehensive quality, and it provides a new way of thinking for the evaluation of college students' comprehensive qualities. It can better overcome the subjective factors of people and make the overall quality evaluation more scientific and reasonable. In this paper, an improved algorithm is used to optimize the BP neural network, and a classroom teaching quality evaluation model based on an improved BP neural network is established. Experimental results show that this method greatly improves the convergence speed of the network and the network results are better.

\section{References}

Bai, S., Li, S., Feng, R., \& Guo, Y. (2010). Organizational project selection based on fuzzy multi-index evaluation and BP neural network. International Conference on Management and Service Science. IEEE, 1 5. http://dx.doi.org/10.1109/ICMSS.2010.5576020

Croft, A. C., Danson, M., Dawson, B. R., \& Ward, J. P. (2001). Experiences of using computer assisted assessment in engineering mathematics. Computers \& Education, 37(1), 53-66. http://dx.doi.org/10.1016/S0360-1315(01)00034-3

Guo, L., Gao, J., Yang, J., \& Kang, J. (2009). Criticality evaluation of petrochemical equipment based on fuzzy comprehensive evaluation and a bp neural network. Journal of Loss Prevention in the Process Industries, 22(4), 469-476. http://dx.doi.org/10.1016/j.jlp.2009.03.003

Rebuffoscheer, C. A., Schmitt, J., Scherer, S. (2007). Differentiation of Listeria monocytogenes Serovars by 
Using Artificial Neural Network Analysis of Fourier-Transformed Infrared Spectra. Appl Environ Microbiol, 73(3), 1036-1040. http://dx.doi.org/ 10.1128/AEM.02004-06

Sun, N., Bai, H., Geng, Y., \& Shi, H. (2017). Price evaluation model in second-hand car system based on BP neural network theory. IEEE/ACIS International Conference on Software Engineering, Artificial Intelligence, NETWORKING and Parallel/distributed Computing. IEEE Computer Society, 431-436. http://dx.doi.org/10.1109/SNPD.2017.8022758

Tinto, V. (1975). Dropout from higher education: a theoretical synthesis of recent research. Review of Educational Research, 45(1), 89-125. https://doi.org/10.3102/00346543045001089

Wang, Y. S., Shen, G. Q., \& Xing, Y. F. (2014). A sound quality model for objective synthesis evaluation of vehicle interior noise based on artificial neural network. Mechanical Systems \& Signal Processing, 45(1), 255-266. http://dx.doi.org/10.1016/j.ymssp.2013.11.001

Zeng, X. H., Guang-Han, L. I., Song, D. F., Sheng, L. I., \& Zhu, Z. C. (2017). Rollover warning algorithm based on genetic algorithm-optimized bp neural network. Journal of South China University of Technology, 45(2), 30-38.

Zhang, N., \& Shen, X. (2009). System identification of tracking error and evaluation of tracking performance using BP neural network. International Symposium on Photoelectronic Detection and Imaging 2009: Advances in Infrared Imaging and Applications. International Society for Optics and Photonics, 7383, 73832F-73832F-9. http://dx.doi.org/10.1117/12.834237 